\title{
The GeoRSS Model and the Design of a Ranking Algorithm in Semantic Web
}

\author{
Anas Bennani, Samira Khoulji, Kerkeb Mohamed Larbi \\ Information Systems Engineering Research Group, Dept. Telecommunications. \\ National School of Applied Science of Tetouan, \\ University Abdelmalek Essaadi, Morocco; \\ anasbenn@gmail.com; khouljisamira@gmail.com; kerkenml@gmail.com
}

\begin{abstract}
E-commerce has become nowadays an influential actor in the international trade industry and has greatly contributed in the economic development of nations. The fact of dealing with this field along with the evolution of technologies renders the e-commerce more usable by internet users. The semantic web offers advanced technical solutions relying on the structuring and organization of the existing data on the web.

Throughout this Paper, I will tackle the issue of e-commerce in relation to geolocation, while at the same time offering the internet user more freedom to detect the surface of the geographical area where he/she is located by a proposing a new geographical coordinate classification algorithm.
\end{abstract}

Keywords-E-commerce, semantic web, Algorithm for Ranking, GEORSS model, geo owl,OGC,FOAF ontology, GOODRELATIONS, geographic information.

\section{Introduction}

E-commerce is defined as the sale or delivery of goods and services by information networks and methods specifically designed for receiving or making orders. This is the definition given by the World Trade Organization (WTO); an international body that deals with the rules of trade between nations. Internet commerce has become essential for many countries of the world. In 2016, French consumers spent 72 billion euros online, which is an increase of 14.6 percent compared to the situation in the previous year (2015). They carried out 103 billion online transactions, equivalent to 33 orders per second, growing by $23 \%$, according to the data published recently on Thursday 26 January by the French Federation of Ecommerce and Distance Selling (FEVAD).

To complete the buying / selling process online, all the procedures, from the product's exposure to its payment, have to be followed in an effective and secure way. Products have to be well described. And their announced prices and the after-sales service have to be well-explained. As long as the products are properly presented on thecompany portal,they will become clearly visible to whoever clients looking for them.

The technology which allows the structuring of data on the web and a better organization is the semantic web. In this regard, Tim Berners Lee has demonstrated his idea by stating the following: "building a web 
of data that might be directly and indirectly processed by the machines so as to help their users to create new knowledge [1] ".

In order to create data in the semantic web, we mainly use an ontology [2]. It is a formulation of a concept. As to our Semantic Web area, the World Wide Web Consortium (W3C) has defined ontology as follows: "An ontology is a formal specification of a common set of terms used to depict and represent an area. It defines the terms used to describe and represent an area of knowledge". [3]

In parallel, the present paper will be concerned with the geographic information System (GIS) that has become a constituent part of our daily lives. And this paper will especially make use of the GIS in the GoodRelations ontology: e-commerce ontology. The GIS is used, as a case in point, to specify the location of a new boutique or a particular store in favor of a company or a trader. More recently, the growth and use of (GIS) were exposed by the implementation of the Web-based tools. The use of geographic information has greatly increased; however, many users will be largely oblivious to the role it plays in their activities. This is because GIS per se is considered as a fairly rare target; rather, it normally creates the scenario under which the topic of interest is remarkable. [4]

The main goal of the present paper is to come up with a new approach of using GoodRelations with the GEORSS model. The latter allows the representation of geographic information on the semantic web. The rationale behind choosing this process that I will study throughout the present paper is to offer any person or tourist the possibility to determine a distance over which he/she may have information about the stores or shops that surround him/her. I suggest that the owners or sellers have to make use of the GoodRelations ontology to present their products and, in the same vein, should make sure that their geographic coordinates are implemented via the properties dedicated to the geolocation. The method I propose here plays an indispensable role in the tourism industry, especially for the benefit of tourists who want to travel around a city without a guide.

\section{Existing Ranking Methods}

\subsection{Spatial data model: GeoRSS}

The Geographically Encoded Objects for RSS feeds (GeoRSS) is a model recommended by the World Wide Web Consortium (W3C). It represents a reference vocabulary for the description of the geo-spatial properties of Web resources. The following illustration shows the UML modelling of GeoRSS. This paper will examine the box-sizing property of the model and will evolve its functionality [5]. 


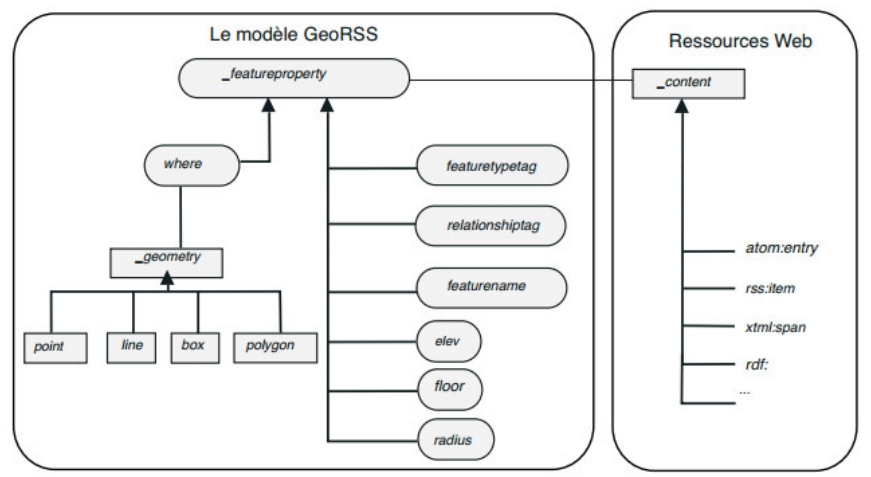

Figure 1: An Extract of the GeoRSS model

The Geography Markup Language (GML) is an improved version of the GeoRSS model. It is an XML grammar for encoding, manipulating and exchanging spatial data. This standard is developed by the Open Geospatial Consortium (OGC) to ensure the interoperability of data, particularly in the fields of Geographic Information Systems and Geomatics. Such sort of language, namely (XML), covers various types of objects which describe spatial data, including entities, coordinate reference systems, geometries, topologies, time, units of measurement and generalized elements [6]. GML is a set of XML schemas that specify an open interchange format for exchanging data and that permit the building of specific models for specialized domains, such as urban management.

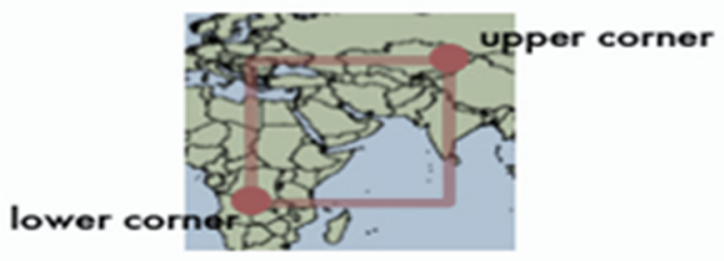

Figure 11: The box-sizing property of the GeoRSS model

The following section will cover the benefits and the added value of this item to the geo-location and its impact on e-commerce.

\subsection{GoodRelations Ontology For E-commerce}

The GoodRelations ontology covers a comprehensive set of procedures dedicated to e-commerce, starting from the description of products until payment. This vocabulary is developed by adding extra semantic codes on Web content [7].

In the present work, I will deal with the issue of e-commerce vis-a-vis the geographic location of stores. The following illustration [7] technically shows the properties of the gr:location class.

\begin{tabular}{|l|}
\hline \multicolumn{1}{|c|}{ gr:Location } \\
\hline gr:name rdfs:Literal \\
gr:description rdfs:Literal \\
gr:hasGlobalLocationNumber xsd:string \\
gr:hasISICv4 xsd:int \\
gr:category rdfs:Literal \\
\hline
\end{tabular}


The objective is to suggest a new way that could allow one to choose a distance that will enable one to delimit the geographical zone where one is located. The desired distance has a direct impact on the surface zone, i.e., if the distance is vast, the surface zone will also be large. Subsequently, thanks to the GoodRelations ontology, one may recognize the various stores located in the area as well as their features and some information on the products that are for sale.

\subsection{Friend of a Friend (FOAF) Ontology ForPerson}

Friend Of a Friend (FOAF) is a widely used ontology in the semantic web that allows to describe people by providing all the available information on them, such as their Web pages, surnames and names, etc. Additionally, this vocabulary offers the possibility to know the relationships they have with each other[8].

In this case study, the ontology will have information about the people who may become customers. In this regard, the storeowners who are located in the delimited geographical area can use that information to capture those people's attention. As a case in point, "a person loves eating chocolate": such information is semantically recorded in the ontology code. In that, if a store, for instance, has chocolate pieces on sale, that person will be automatically notified of the offer.

\section{The Spatial Datum in the Semantic Web}

\subsection{Determination of GPS Coordinates.}

In order to give importance to the geographical location of a particular person, I suggest an approach which will improve the detection of the geographical area. To this end, we can use the GeoRSS model that we have presented in the above-mentioned chapter under the property "Box". The suggested scenario is the following:

The person who may become a customer with a store is found in a location specified by the geographic latitude and longitude coordinates (Lat1\& Lon1). These parameters represent the first coordinates "lower corner" of the Box. The added value of this item is summarized as follows: so as to establish the geographic coordinates (Lat2 \& Lon 2) of the "upper Corner" of the Box, one could determine the distance at which one may be surrounded by the rectangle traced by the property Box, with an orientation equal to $45^{\circ}=$ $\mathrm{Pi} / 4$ Radians. Normally, if we are to detect the geographic coordinates of a particular destination, we will need to know the coordinates of the departure, of the distance covered and of the orientation angle. Since its parameters are known, we make use of the formula developed by Thaddeus Vincenty[9] to find those coordinates following an ellipsoidal model of the Earth.

Lat2 $=\operatorname{ASIN}(\operatorname{SIN}($ lat1 $) * \operatorname{COS}(\mathrm{d} / \mathrm{R})+\operatorname{COS}($ lat 1$) * \operatorname{SIN}(\mathrm{d} / \mathrm{R}) * \cos ($ Orient $))$

Lon2 = lon1 + ATAN2 $(\operatorname{COS}(\mathrm{d} / \mathrm{R})-\operatorname{SIN}($ lat1 $) * \operatorname{SIN}($ lat2 $), \operatorname{SIN}($ Orient $) * \operatorname{SIN}(\mathrm{d} / \mathrm{R}) * \operatorname{COS}($ lat1) $)$

Knowing that R: Ray of the Earth $=6371 \mathrm{Km}$; $d=$ distance in Kilometers $(\mathrm{Km})$; Orient $=$ Orientation in radians. 


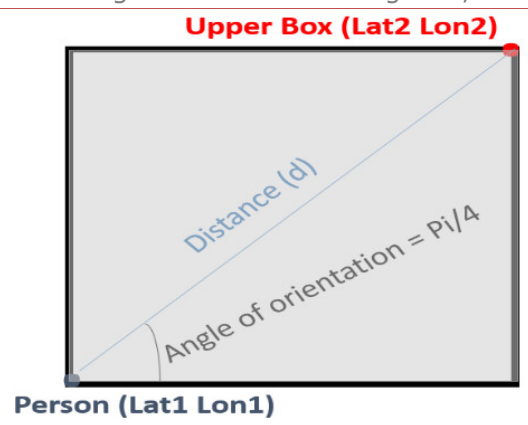

Figure 13: Setup of the Property Box

The GeoRSS -Simple model makes use of the World Geodetic System (WGS84). Geodesy, in this respect, is a coordinate system used to indicate the trajectory corresponding to the minimum distance between two reference points on a surface. As to the sphere, it is the arc of a great circle. For instance, by knowing the position of two points represented in $A$ and $B$ on a sphere, we could, therefore, so as to estimate the distance between them, calculate the curvilinear abscissa $S(A B)$ on the great circle passing through $A$ and $B$, as it is shown in the following figure. Geodesy is the science that studies the Earth's geometric shape, dimensions and gravity field. Its main objective is to develop terrestrial reference systems to which any georeferenced data user or creator can have access through the intermediary network.

The Adoption and implementation of such reference systems constitute an essential standardization tool for the geographic information in particular and the positioning in general. Nonetheless, the calculations of a spherical model are accurate to within $0.3 \%$. Vincenty's formula is accurate to within $0.5 \mathrm{~mm}$ or 0.000015 'on the ellipsoid used. Note that the accuracy of theWGS-84 model will be lower according to the Earth's position, but generally in the range of $0.003 \%[10]$.

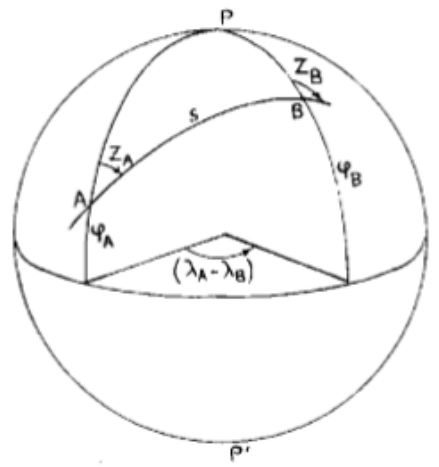

Figure 14: The Position of Two Points on a "Geodesic" Sphere

\subsection{Algorithm Conditions for the Ranking of Geographic Datum}

The algorithm that is proposed for this item requires conditions at the organizational level. Admittedly, the author of this paper had a Geographic Coordinate Database which included the stores- in ascending order- of the region or the city where he was located. This starts with the latitude and, then, longitude coordinates.

This paper considers that those coordinates are gathered in a semantic way thanks to the GoodRelations ontology and are sorted out in ascending order and stored in a table. Here is an example: 
Table 13: An Example of sorting out the geographic coordinates of stores

\begin{tabular}{c|cc} 
& LATITUDE & LONGITUDE \\
\hline STORE 1 & 0,7673214 & 0,0236234 \\
STORE 2 & 0,7673300 & 0,0236261 \\
STORE 3 & 0,7673416 & 0,0236125 \\
STORE 4 & 0,7673675 & 0,0236259 \\
STORE 5 & 0,7673780 & 0,0236395 \\
STORE 6 & 0,7673865 & 0,0237264 \\
STORE 7 & 0,7673868 & 0,0227456 \\
\hline...... & &
\end{tabular}

As far as the programming language is concerned, the coordinates of the store 4 will be stored in a table as follows:

Latitude $=\operatorname{Tab}[3][0]=0.7673675$ and Longitude $=$ Tab [3] [1] $=0.0236259$.

\subsection{Algorithm}

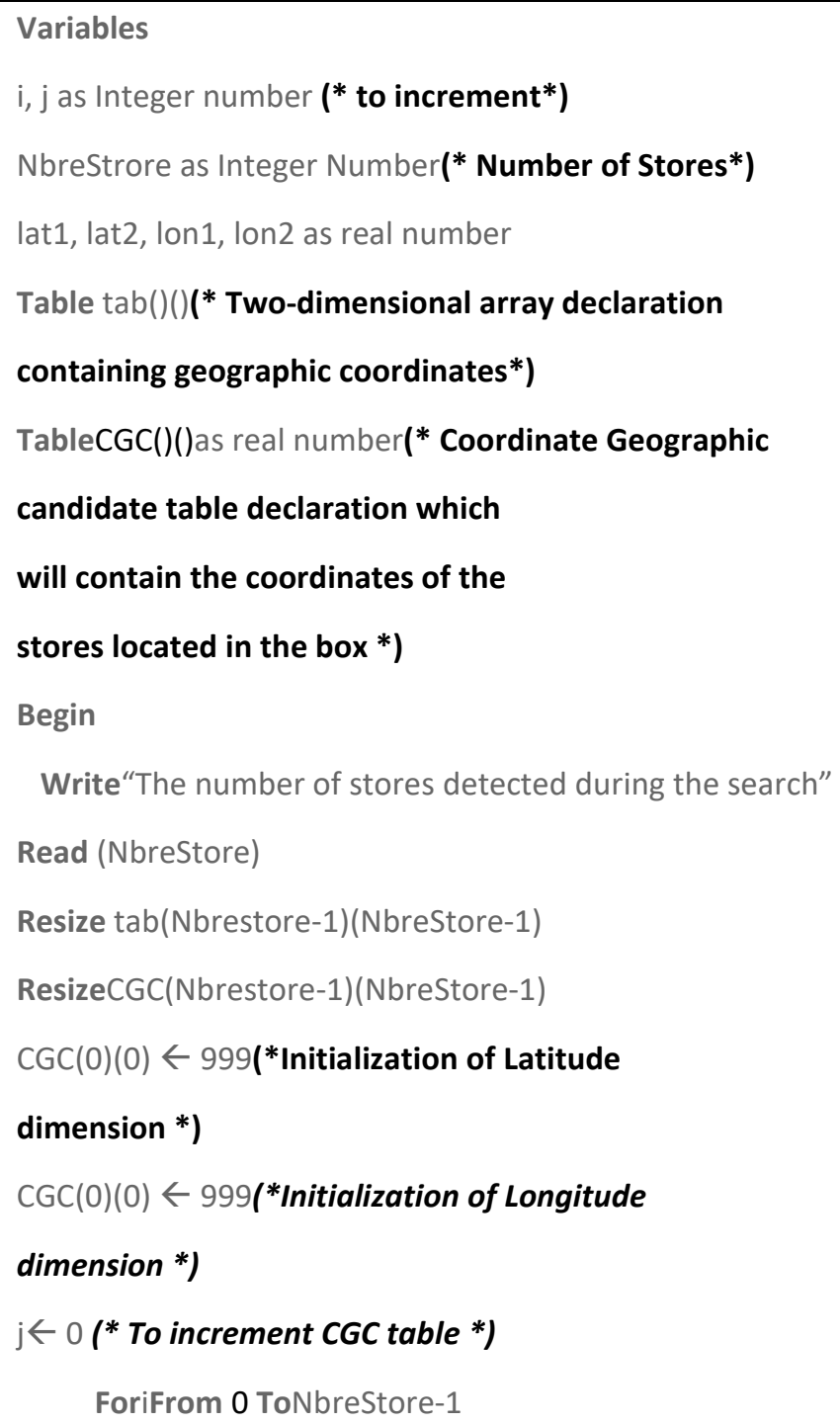




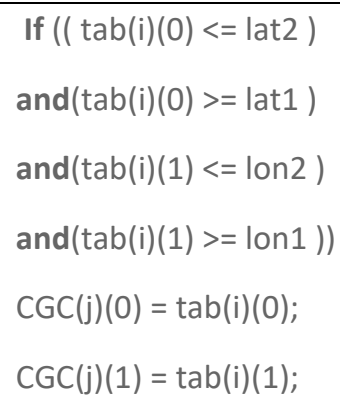

\subsection{The Positioning of stores in relation to a particular person}

Once the geographic coordinates of the listed stores pertain to the surface of the Box via the algorithm, we could recognize the distance between the store and the position of the person requesting the information, as well as the orientation (Angle to the North). And this is thanks to the following formulae: $\mathbf{d}=\operatorname{ACOS}(\operatorname{SIN}($ lat 1$) * \operatorname{SIN}($ lat2 $)+\cos ($ lat1 $) * \cos ($ lat2 $) * \cos ($ lon2-lon 1$)) * \mathrm{R}$

Orientation $=\operatorname{ATAN} 2(\operatorname{COS}($ lat 1$) * \operatorname{SIN}($ lat2 $)-\operatorname{SIN}($ lat1 $) * \cos ($ lat2 $) * \cos ($ lon2-lon1 $), \operatorname{SIN}($ lon2lon 1$) * \cos ($ lat2 $))$ Here follows a mathematically equivalent formula which is, however, less prone to errors of a rounding nature as far the short distances are concerned:

$\mathbf{d}=2^{*} \operatorname{asin}\left(\operatorname{sqrt}\left((\sin ((\text { lat1-lat2 }) / 2))^{\wedge} 2+\cos (\text { lat1 })^{*} \cos (\text { lat2 })^{*}(\sin ((\operatorname{lon} 1-\operatorname{lon} 2) / 2))^{\wedge} 2\right)\right)$

Such formulas are set for a spherical model of the Earth and constitute, as a result, the approximations. [11]

\section{Conclusion}

In this research paper, I have, on the one hand, shown interest in e-commerce and its link with the spatial dimension. The goal, as mentioned earlier, is to offer internet users, generally, the opportunity to conduct the desired search on the geographical area where they are located and to enable them to obtain as much information as possible.

The fact of combining e-commerce with geolocation in a structured manner contributes to a better organization of the data. I have also demonstrated, on the other hand, that e-commerce has become a highly intelligent sector in the sense that it gives all Internet users, who are now attached to technology more than ever before, the possibility to automatically carry out any sophisticated commercial operation with the minimum number of interventions.

The result mentioned in this paper will contribute to the development of the tourist and economic sectors of the countries because it allows people in general and tourists in particular to identify the shops around 
them and know the types of their products offered for salefrom research. This paper also facilitates the sales / buying process either at the seller level by targeting buyers who are interested in their products, or aside from the buyer who finds the desired products that he will be able to buy them and at the minimum of time.

\section{REFERENCES}

[1] Tim Berners-Lee, Book: “Weaving The Web”, 1999

[2] Grigoris Antoniou, Frank Van Harmelen, "A Semantic Web Primer", Fltebook, The Mit Press Cambridge Massachusetts, London, England 2004.

[3] Liyang Yu, “A Developer Guide To The Semantic Web”, Springer, Isbn : 9783642159695,Pp. 136,137, 2011.

[4] Glen Hart,Catherine Dolbear, Ebook “Linked Data A Geographic Perspective”, Isbn: 13: 978-1-4398-69970, 2013.

[5] Wafa Mefteh, Thesis : “An Ontological Approach For Modeling And Reasoning On Trajectories: Taking Into Account The Thematic, Temporal And Spatial Aspects", The University - La Rochelle -2013

[6] G. Percivall, "Opengis Reference Model", Document Number Ogc 03-040,Version 0.1.3. Open Gis Consortium, Inc., 2003.

[7] Martin Hepp, Official Web Site Http://Www.Heppnetz.De/Projects/Goodrelations/

[8] Official Web Site Of Foaf Ontology $\mathrm{Http}: / /$ Www.Foaf-Project.Org

[9] T. Vinccnty, "Direct And Inverse Solutions Of Geodesics On The Ellipsoid WIth Application Of Ested Equations", Directorate Of Overseas Surveys Of The Ministry Of Overseas Developmen, 1975.

[10] Official Web Site Of National Institute Of Geographic And Forestry Information http://Geodesie.Ign.Fr

[11] H. P. Garnir, Institute Of Nuclear, Atomic And Spectroscopic Physics, "Calculation Of The Distance Between Two Points", University Of Liège Belgium, Training Day At The University Of Liège, 2004 